\title{
A Fixed-loaded Interface Component Mode Synthesis Method for Dynamic Analysis of a Folding Wing
}

\author{
Yingge $\mathrm{Ni}^{1, \mathrm{a}}$, Xiaopeng Wan ${ }^{2, \mathrm{~b}^{*}}$, Meiying Zhao ${ }^{3, \mathrm{c}}$ \\ 1, 2, 3 School of Aeronautics, Northwestern Polytechnical University, Xi'an 710072, China \\ aygni.good@163.com, bwanxp@nwpu.edu.cn, ${ }^{\mathrm{c}}$ zhaomeiying@nwpu.edu.cn
}

Keywords: Folding wing, Component mode synthesis, Model reduction, Dynamic analysis

\begin{abstract}
A fixed-loaded interface component mode synthesis method is presented for carrying out a dynamic analysis for a folding wing. The present method has the advantages of the fixed interface method and loaded interface method, in which the effects of the elastic and inertia force at interfaces are included and the size of dynamic problem is highly reduced through elimination of interface coordinates. It is shown in the paper that the present method is able to predict the natural frequency accurately. Further, it can be integrated with the multibody dynamic code to perform the transient dynamic analysis during the morphing process.
\end{abstract}

\section{Introduction}

Morphing aircrafts, which could be capable of substantially changing their shapes during flight to enable a single vehicle to perform multiple mission roles, draw future aircraft designers' attention. One strong candidate for morphing aircraft design is a folding wing configuration [1-2]. The rapid changes in the wing shape will raise complex problems during the large-scale morphing process. So extensive studies on aeroelastic problem of a folding wing have been performed over the past decade [3-8]. For example, Reich和Scarlett [3-4] carried out the aeroelastic multibody morphing simulation of a folding wing through integration of finite element modeling using fixed interface synthesis, aerodynamic loading, and continuous-time multibody simulation. Liu [5] performed a reduced-order aeroelastic models in time space in order to achieve fast aeroelastic analysis via structural FEM reduced-order modeling and aerodynamics reduced-order modeling. Selitrennik [6] presented an aeroelastic simulation of rapid morphing flight vehicles, in which a time-dependent coordinate transformation matrix was used to perform the fictious-mass modal synthesis. Zhao and $\mathrm{Hu}^{[7-8]}$ established a set of completely structural equations of motion that govern the time evolutions of the folding wing during the morphing process through the component synthesis.

Among these studies, an evident feature of the structural modeling is the component mode synthesis (CMS). The detailed finite element model of a folding wing typically has hundreds of thousands degrees of freedom, namely mass and stiffness matrices of large order. To retain such large matrices to perform the analysis may be practically impossible. At the same time, correctives or modifications of the structure as a whole usually fail. To address above challenges, CMS is a natural choice. It enables the dynamic analysis to be performed on highly reduced matrices to save computational time. Also, it can make modifications possible to reduce the number of design iterations [9-11].

Since Hurty[12] introduced first is a fixed interface component synthesis. Many modified versions referred to it have been reported. Craig and Bampton[10] used the normal vibration and constrained modes to describe the dynamic behavior. Suares and Singh[13] presented a higher order modal combination method to accelerate the convergence of coupling process. Rixen[14] developed a dual fixed interface method, in which the interface forces and the residual flexibility matrix were taken into consideration. However, the final equation includes the interface coordinates. When the interface has many freedoms, it can not contribute to reduce the size of the equation. Benfield and Hruda[15] proposed a loaded interface method, in which the stiffness and mass at interface were under 
consideration. Lim et al [16] demonstrated a modified interface method, in which the normal vibration modes included the effects of the elastic and inertial force applied at the interfaces.

In this paper, a modified interface component mode synthesis of a folding wing is proposed based on fixed interface and loaded interface method. The continuity at the interface is used to couple each component. The final dynamic equation has a highly reduced size and more concise. To verify the accuracy of this method, the results are compared with those from MSC.NASTRAN. In addition, this method can hold true for more complex structures.

\section{Fixed-loaded interface component mode synthesis for a folding wing}

The folding wing consists of three separate components: the fuselage, the inboard wing and the outboard wing, denoted A, B, C, respectively. All components are modeled by plates with the Yong's modulus of $7.1 \times 10^{10} \mathrm{~Pa}$, Poisson's coefficient of 0.33 and a density of $2.7 \times 10^{3} \mathrm{~kg} / \mathrm{m}^{3}$, which are discretized using CQUAD4 elements with 2mm thickness in MSC.NASTRAN. Each node of this element has six degrees of freedom: three translations, namely, $u_{x}, u_{y}, u_{z}$ and three rotations, i.e., $\theta_{x}, \theta_{y}, \theta_{z}$.For each component, all nodes at the interface are used to coordinate the interface displacement. In addition, hinges between components are model as a set of torsional spring at several points and MPC. The rotational degree of freedom $\theta_{x}$ is coupled using torsional springs while the other five degrees of freedom are defined via MPC. For brevity, all freedoms at the interfaces are coupled with MPC. The finite element model and coordinate systems for a folding wing are shown in Fig.1.

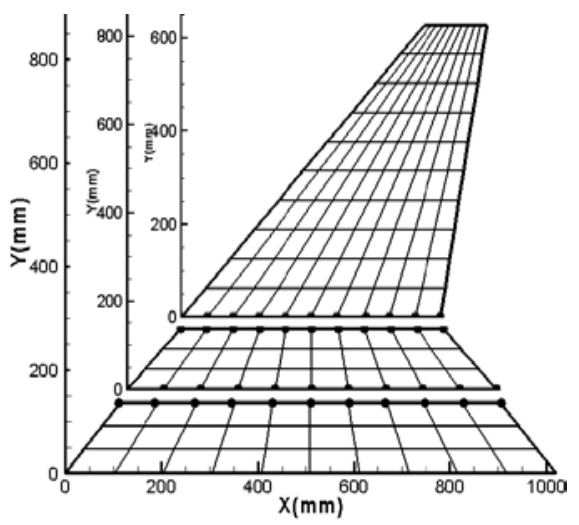

(a) Finite element model of folding wing

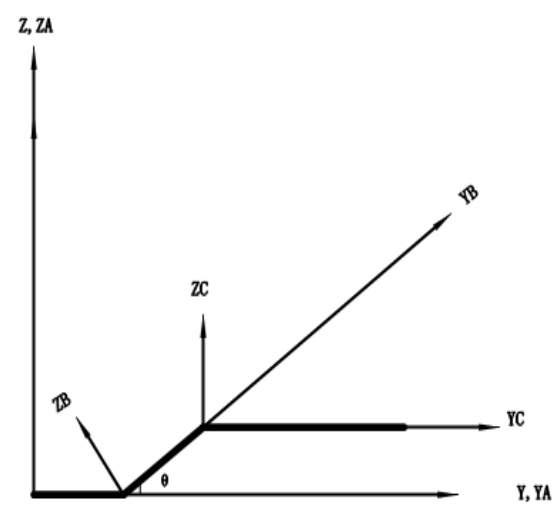

(b) Coordinate systems

Fig.1 Finite element model of folding wing and coordinate systems (each circle represents the hinge position)

The dynamic equation of each component with no damp is written in local coordinates as follows: $\mathbf{M}^{\lambda} \dot{\mathbf{i}}^{\lambda}+\mathbf{K}^{\lambda} \mathbf{u}^{\lambda}=\mathbf{f}^{\lambda},(\lambda=A, B, C)$

where $\mathbf{M}^{\lambda}$ and $\mathbf{K}^{\lambda}$ stand for mass and stiffness matrices. $\mathbf{u}^{\lambda}$ is a displacement vector. $\mathbf{f}^{\lambda}$ is the constraints force vector.

To facilitate the application of the component mode synthesis, the dynamic equation of each component can be partitioned as in the global coordinate:

$\left[\begin{array}{ll}\mathbf{M}_{i i}^{A} & \mathbf{M}_{i j}^{A} \\ s y m & \mathbf{M}_{j j}^{A}\end{array}\right]\left[\begin{array}{c}\ddot{\mathbf{u}}_{i}^{A} \\ \ddot{\mathbf{u}}_{j}^{A}\end{array}\right]+\left[\begin{array}{cc}\mathbf{K}_{i i}^{A} & \mathbf{K}_{i j}^{A} \\ s y m & \mathbf{K}_{j j}^{A}\end{array}\right]\left[\begin{array}{c}\mathbf{u}_{i}^{A} \\ \mathbf{u}_{j}^{A}\end{array}\right]=\left[\begin{array}{c}\mathbf{0} \\ \mathbf{f}_{j}^{A}\end{array}\right]$

$\left[\begin{array}{ccc}\mathbf{M}_{i i}^{B} & \mathbf{M}_{i j}^{B a} & \mathbf{M}_{i j}^{B b} \\ & \mathbf{M}_{j j}^{B a} & \mathbf{M}_{j j}^{B a b} \\ s y m & & \mathbf{M}_{j j}^{B b}\end{array}\right]\left[\begin{array}{c}\ddot{\mathbf{u}}_{i}^{B} \\ \ddot{\mathbf{u}}_{j}^{B a} \\ \ddot{\mathbf{u}}_{j}^{B b}\end{array}\right]+\left[\begin{array}{ccc}\mathbf{K}_{i i}^{B} & \mathbf{K}_{i j}^{B a} & \mathbf{K}_{i j}^{B b} \\ & \mathbf{K}_{j j}^{B a} & \mathbf{K}_{j j}^{B a b} \\ s y m & & \mathbf{K}_{j j}^{B b}\end{array}\right]\left[\begin{array}{c}\mathbf{u}_{i}^{B} \\ \mathbf{u}_{j}^{B a} \\ \mathbf{u}_{j}^{B b}\end{array}\right]=\left[\begin{array}{c}\mathbf{0} \\ \mathbf{f}_{j}^{B a} \\ \mathbf{f}_{j}^{B b}\end{array}\right]$

$\left[\begin{array}{ll}\mathbf{M}_{i i}^{C} & \mathbf{M}_{i j}^{C} \\ s y m & \mathbf{M}_{j j}^{C}\end{array}\right]\left[\begin{array}{c}\ddot{\mathbf{u}}_{i}^{C} \\ \ddot{\mathbf{u}}_{j}^{C}\end{array}\right]+\left[\begin{array}{cc}\mathbf{K}_{i i}^{C} & \mathbf{K}_{i j}^{C} \\ s y m & \mathbf{K}_{j j}^{C}\end{array}\right]\left[\begin{array}{c}\mathbf{u}_{i}^{C} \\ \mathbf{u}_{j}^{C}\end{array}\right]=\left[\begin{array}{c}\mathbf{0} \\ \mathbf{f}_{j}^{C}\end{array}\right]$ 
where the mass and stiffness matrices are partitioned according to the interior displacements (i), the interface displacements $(\mathrm{j})$. $a$ represents the interface between components A and B. $b$ donates the interface between components B and C.

\section{Fixed interface component formulation}

For component A and C, the displacements are the summation of normal mode and constrained mode. Normal modes are obtained from the eigenvalue analysis:

$\left(\mathbf{K}_{i i}^{A}-\omega^{A 2} \mathbf{M}_{i i}^{A}\right) \boldsymbol{\varphi}_{n}^{A}=\mathbf{0},\left(\mathbf{K}_{i i}^{C}-\omega^{C 2} \mathbf{M}_{i i}^{C}\right) \boldsymbol{\varphi}_{n}^{C}=\mathbf{0}$

where $\boldsymbol{\varphi}_{n}^{A}, \boldsymbol{\varphi}_{n}^{C}$ is the normal mode matrix and $\omega$ is the natural frequency. Further, the normal modes are truncated. Only the required numbers of modes are included in order to reduce the size of problem.

Constrained modes are obtained from the static analysis. The linear relationship between the forces and displacements with stiffness matrix are as follows:

$\left[\begin{array}{ll}\mathbf{K}_{i i}^{A} & \mathbf{K}_{i j}^{A} \\ \operatorname{sym} & \mathbf{K}_{j j}^{A}\end{array}\right]\left[\begin{array}{c}\mathbf{u}_{i}^{A} \\ \mathbf{u}_{j}^{A}\end{array}\right]=\left[\begin{array}{c}\mathbf{0} \\ \mathbf{f}_{j}^{A}\end{array}\right],\left[\begin{array}{cc}\mathbf{K}_{i i}^{C} & \mathbf{K}_{i j}^{C} \\ s y m & \mathbf{K}_{j j}^{C}\end{array}\right]\left[\begin{array}{c}\mathbf{u}_{i}^{C} \\ \mathbf{u}_{j}^{C}\end{array}\right]=\left[\begin{array}{c}\mathbf{0} \\ \mathbf{f}_{j}^{C}\end{array}\right]$

Since there is no force acting on the interior displacements. From the first row of the equation above, one obtains

$\mathbf{u}_{i}^{A}=-\left(\mathbf{K}_{i i}^{A}\right)^{-1} \mathbf{K}_{i j}^{A} \mathbf{u}_{j}^{A}=\boldsymbol{\varphi}_{c}^{A} \mathbf{u}_{j}^{A}, \mathbf{u}_{i}^{C}=-\left(\mathbf{K}_{i i}^{C}\right)^{-1} \mathbf{K}_{i j}^{C} \mathbf{u}_{j}^{C}=\boldsymbol{\varphi}_{c}^{C} \mathbf{u}_{j}^{C}$

where $\boldsymbol{\varphi}_{c}^{A}, \boldsymbol{\varphi}_{c}^{C}$ defines the constrained modes of component A and C. So the displacement can be expressed as:

$\left[\begin{array}{c}\mathbf{u}_{i}^{A} \\ \mathbf{u}_{j}^{A}\end{array}\right]=\left[\begin{array}{cc}\boldsymbol{\varphi}_{n}^{A} & \boldsymbol{\varphi}_{c}^{A} \\ \mathbf{0} & \mathbf{I}\end{array}\right]\left[\begin{array}{l}\mathbf{p}^{A} \\ \mathbf{u}_{j}^{A}\end{array}\right],\left[\begin{array}{c}\mathbf{u}_{i}^{C} \\ \mathbf{u}_{j}^{C}\end{array}\right]=\left[\begin{array}{cc}\boldsymbol{\varphi}_{n}^{C} & \boldsymbol{\varphi}_{c}^{C} \\ \mathbf{0} & \mathbf{I}\end{array}\right]\left[\begin{array}{l}\mathbf{p}^{C} \\ \mathbf{u}_{j}^{C}\end{array}\right]$

Here, the size of problem is reduced through the mode truncation while the interface displacements are still kept.

\section{Loaded interface component formulation}

In the following is the deduction of loaded interface method for component B. The compatibility equations can be expressed as:

$\mathbf{u}_{j}^{A}=\mathbf{u}_{j}^{B a}, \mathbf{u}_{j}^{C}=\mathbf{u}_{j}^{B b}$

Here, the total constrained motion of component $\mathrm{A}$ and $\mathrm{C}$ can be expressed in another form:

$\left[\begin{array}{c}\mathbf{u}_{i}^{A} \\ \mathbf{u}_{j}^{A}\end{array}\right]=\left[\begin{array}{c}\boldsymbol{\varphi}_{c}^{A} \\ \mathbf{I}\end{array}\right] \mathbf{u}_{j}^{A},\left[\begin{array}{c}\mathbf{u}_{i}^{C} \\ \mathbf{u}_{j}^{C}\end{array}\right]=\left[\begin{array}{c}\boldsymbol{\varphi}_{c}^{C} \\ \mathbf{I}\end{array}\right] \mathbf{u}_{j}^{C}$

Substituting the Eq.(9) into Eq.(10), it becomes

$\mathbf{u}^{A}=\left[\begin{array}{c}\mathbf{u}_{i}^{A} \\ \mathbf{u}_{j}^{A}\end{array}\right]=\left[\begin{array}{c}\boldsymbol{\varphi}_{c}^{A} \\ \mathbf{I}\end{array}\right]\left[\begin{array}{lll}\mathbf{0} & \mathbf{I} & \mathbf{0}\end{array}\right]\left[\begin{array}{c}\mathbf{u}_{i}^{B} \\ \mathbf{u}_{j}^{B a} \\ \mathbf{u}_{j}^{B b}\end{array}\right]=\mathbf{T}_{1} \mathbf{u}^{B}, \quad \mathbf{u}^{C}=\left[\begin{array}{c}\mathbf{u}_{i}^{C} \\ \mathbf{u}_{j}^{C}\end{array}\right]=\left[\begin{array}{c}\boldsymbol{\varphi}_{c}^{C} \\ \mathbf{I}\end{array}\right]\left[\begin{array}{lll}\mathbf{0} & \mathbf{0} & \mathbf{I}\end{array}\right]\left[\begin{array}{c}\mathbf{u}_{i}^{B} \\ \mathbf{u}_{j}^{B a} \\ \mathbf{u}_{j}^{B b}\end{array}\right]=\mathbf{T}_{2} \mathbf{u}^{B}$

Substituting Eq.(11) into Eqs.(2) and (4), and premultiplying by $\mathbf{T}_{1}^{T}$ and $\mathbf{T}_{2}^{T}$,respectively. one obtains:

$\overline{\mathbf{M}}^{A} \ddot{\mathbf{u}}^{B}+\overline{\mathbf{K}}^{A} \mathbf{u}^{B}=\overline{\mathbf{f}}^{A}, \quad \overline{\mathbf{M}}^{C} \ddot{\mathbf{u}}^{B}+\overline{\mathbf{K}}^{C} \mathbf{u}^{B}=\overline{\mathbf{f}}^{C}$

where, $\overline{\mathbf{M}}^{A}=\mathbf{T}_{1}^{T} \mathbf{M}^{A} \mathbf{T}_{1}, \overline{\mathbf{K}}^{A}=\mathbf{T}_{1}^{T} \mathbf{K}^{A} \mathbf{T}_{1}, \overline{\mathbf{f}}^{A}=\mathbf{T}_{1}^{T} \mathbf{f}^{A}, \overline{\mathbf{M}}^{C}=\mathbf{T}_{2}^{T} \mathbf{M}^{C} \mathbf{T}_{2}, \overline{\mathbf{K}}^{C}=\mathbf{T}_{2}{ }^{T} \mathbf{K}^{C} \mathbf{T}_{2}$, $\overline{\mathbf{f}}^{C}=\mathbf{T}_{2}^{T} \mathbf{f}^{C}$.

The dynamic equation of component B can be easily formed with Eq.(3) and (12). The final result can be expressed as follows:

$\overline{\mathbf{M}}^{B} \ddot{\mathbf{u}}^{B}+\overline{\mathbf{K}}^{B} \mathbf{u}^{B}=\mathbf{0}$

where $\overline{\mathbf{M}}^{B}=\overline{\mathbf{M}}^{A}+\mathbf{M}^{B}+\overline{\mathbf{M}}^{C}, \overline{\mathbf{K}}^{B}=\overline{\mathbf{K}}^{A}+\mathbf{K}^{B}+\overline{\mathbf{K}}^{C}$.

It is easy to show that the dynamic effects at interfaces from component $A$ and $C$ are included. The right-hand side of the above equation equals zeros. The interface forces cancel out when assemble on 
the interface. Therefore, Eq.(13) is a free vibration equations of motion. Eigenvector solution yields the normal mode transformation.

$$
\mathbf{u}^{B}=\boldsymbol{\varphi}^{B} \mathbf{p}^{B}=\left[\begin{array}{c}
\boldsymbol{\varphi}_{i}^{B} \\
\boldsymbol{\varphi}_{j a}^{B} \\
\boldsymbol{\varphi}_{j b}^{B}
\end{array}\right] \mathbf{p}^{B}
$$

where $\boldsymbol{\varphi}^{B}$ is the normal mode of component $\mathrm{B}$ and $\mathbf{p}^{B}$ is the modal displacement. The above equation and the corresponding eigenvalues now represent the modes of component $B$. This type of component modes will enhance the accuracy of the final results. It is noted that all the mass and stiffness of component $\mathrm{A}$ and $\mathrm{C}$ are used without any change in the preceding procedure.

\section{Synthesis of a folding wing}

The next step in the component mode synthesis is the coupling of the equations of each component. The dynamic equation for uncoupled components can be combined in uncoupled form as:

$$
\tilde{\mathbf{M}} \ddot{\mathbf{p}}+\tilde{\mathbf{K}} \mathbf{p}=\tilde{\mathbf{f}}
$$

where $\tilde{\mathbf{M}}=\operatorname{diag}\left(\mathbf{M}^{A}, \mathbf{M}^{B}, \mathbf{M}^{C}\right), \tilde{\mathbf{K}}=\operatorname{diag}\left(\mathbf{K}^{A}, \mathbf{K}^{B}, \mathbf{K}^{C}\right), \tilde{\mathbf{f}}=\left(\mathbf{f}^{A T}, \mathbf{f}^{B T}, \mathbf{f}^{C T}\right)$.

The dynamic equation of each component can be coupled by imposing the compatibility conditions. Here, introducing the coordinate transformation, one obtains:

$$
\left[\begin{array}{c}
\mathbf{u}_{i}^{A} \\
\mathbf{u}_{j}^{A} \\
\mathbf{u}^{B} \\
\mathbf{u}_{i}^{C} \\
\mathbf{u}_{j}^{C}
\end{array}\right]=\left[\begin{array}{ccc}
\boldsymbol{\varphi}^{A} & \boldsymbol{\varphi}_{c}^{A} \boldsymbol{\varphi}_{j a}^{B} & \mathbf{0} \\
\mathbf{0} & \boldsymbol{\varphi}_{j a}^{B} & \mathbf{0} \\
\mathbf{0} & \boldsymbol{\varphi}^{B} & \mathbf{0} \\
\mathbf{0} & \boldsymbol{\varphi}_{c}^{A} \boldsymbol{\varphi}_{j b}^{B} & \boldsymbol{\varphi}^{C} \\
\mathbf{0} & \boldsymbol{\varphi}_{j b}^{B} & \mathbf{0}
\end{array}\right]\left[\begin{array}{l}
\mathbf{p}^{A} \\
\mathbf{p}^{B} \\
\mathbf{p}^{C}
\end{array}\right]=\mathbf{S} \mathbf{p}
$$

As for the coordinate transformation, the submatrix of the normal mode of component $\mathrm{B}$ is substituted for the constrained modes of component A and C. so the interface coordinates vanish. Premultiplying the coordinate transformation $\mathbf{S}^{T}$, the final reduced dynamic equation can be written as follows.

$$
\mathbf{M} \ddot{\mathbf{p}}+\mathbf{K p}=\mathbf{0}
$$

where $\mathbf{M}=\mathbf{S}^{T} \tilde{\mathbf{M}} \mathbf{S}, \mathbf{K}=\mathbf{S}^{T} \tilde{\mathbf{K}} \mathbf{S}$. The force function term $\tilde{\mathbf{f}}$ vanishes. Therefore, the equation above corresponds to the free vibration equations for a folding wing. The natural frequencies of a folding wing can be obtained from the solution of the eigenvalue problem. In the next section the procedure described above is used to analyze the folding wing.

\section{Results and discussion}

To verify the method in this paper, the free vibration of a folding wing is demonstrated. The folding wing is idealized here with 160 elements, 209 nodes and a total of 1254 degrees of freedom. The synthesis is implemented using MATLAB codes. For the synthesis, all nodes at interfaces are used. The number of degrees of freedom at each component's interface is 66.DMAP language is used to extract the mass and stiffness matrices of each component in MSC.NASTRAN. And then, these matrices are partitioned according to the interior and interface freedom. The final equation of motion for a folding wing is established through substructure synthesis described above in this paper.

When the present CMS and fixed interface CMS are used to derive the final equation, the number of mode of the components A, B and C is 15, 15 and 30, respectively. Therefore, the size of the final eigenvalue problem is 60 and 192. Compared with the original size 1284, the present CMS have a great advantage to reduce the size of problem. The corresponding natural frequency of the total folding wing is given in Table 1. 
Table 1 Comparative result of natural frequencies

\begin{tabular}{|c|c|c|c|}
\hline $\begin{array}{l}\text { Mode } \\
\text { order }\end{array}$ & $\begin{array}{c}\text { MSC.NASTRA } \\
\text { N/ Hz }\end{array}$ & $\begin{array}{c}\text { Present } \\
\mathrm{CMS} / \mathrm{Hz}\end{array}$ & $\begin{array}{c}\text { Fixed interface } \\
\mathrm{CMS} / \mathrm{Hz}\end{array}$ \\
\hline 1 & 3.15105 & 3.46063 & 3.46063 \\
\hline 2 & 12.29035 & 12.67006 & 12.67005 \\
\hline 3 & 19.17830 & 20.49874 & 20.49685 \\
\hline 4 & 30.28381 & 30.85593 & 30.85434 \\
\hline 5 & 42.46986 & 45.86567 & 45.86502 \\
\hline 6 & 54.47676 & 55.37355 & 55.35777 \\
\hline 7 & 66.44668 & 66.96086 & 66.94919 \\
\hline 8 & 77.71950 & 78.72086 & 78.68926 \\
\hline 9 & 85.02097 & 86.72906 & 86.70125 \\
\hline 10 & 104. 0730 & 103.98572 & 103.94089 \\
\hline 11 & 118.6697 & 118.95313 & 118.72415 \\
\hline 12 & 123.7177 & 124.63282 & 124.43569 \\
\hline 13 & 127.3209 & 128.63282 & 128.44513 \\
\hline 14 & 149.1986 & 149.70067 & 149.64329 \\
\hline 15 & 166.5269 & 167.24053 & 166.91276 \\
\hline 16 & 172.7446 & 172.86101 & 172.77981 \\
\hline 17 & 189.1777 & 190.70873 & 190.373434 \\
\hline 18 & 192.3538 & 193.13321 & 192.57242 \\
\hline 19 & 207.6614 & 208.96556 & 207.93946 \\
\hline 20 & 221.9039 & 222.48247 & 221.93315 \\
\hline
\end{tabular}

Table 1 also indicates a good agreement between the first twenty natural frequencies computed using the present CMS and those via MSC.NASTRAN. Compared with the result from fixed interface CMS, the natural frequency of present CMS is a little higher. However, the interfaces coordinate are reduced. And the resulting equation is of highly reduced order.
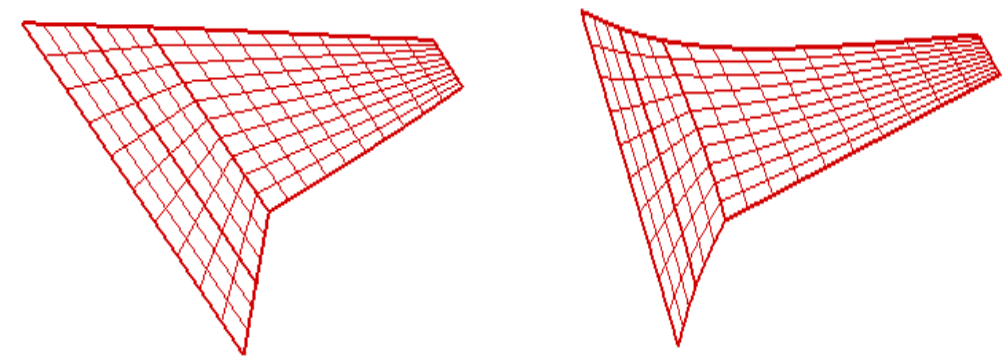

(a) The first mode shape comparison
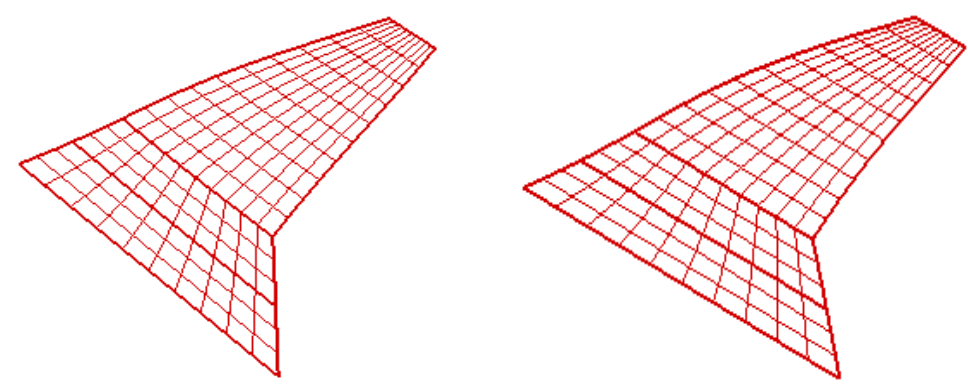

(b) The second mode shape comparison 

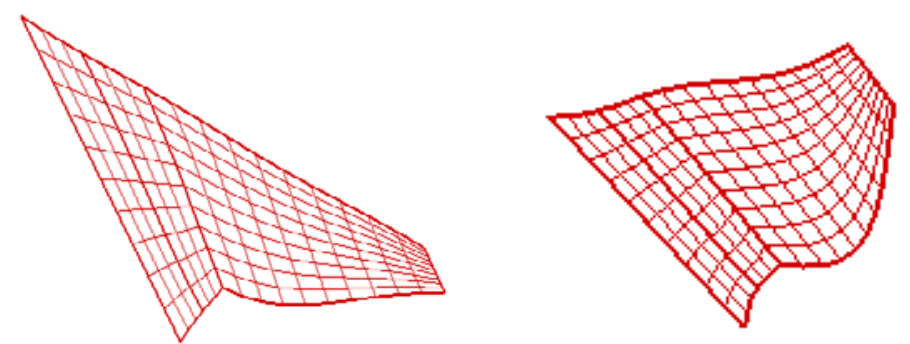

(c) The third mode shape comparison
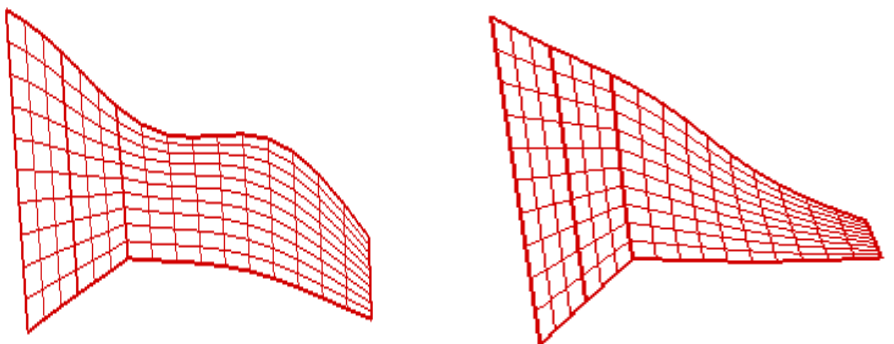

(d) The fourth mode shape comparison
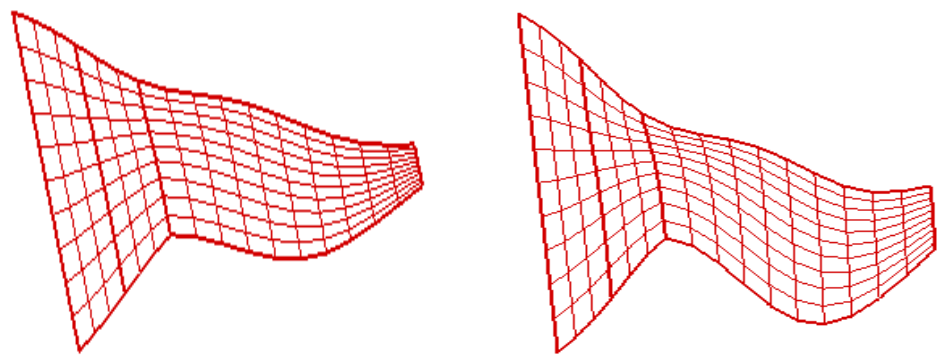

(e) The fifth mode shape comparison
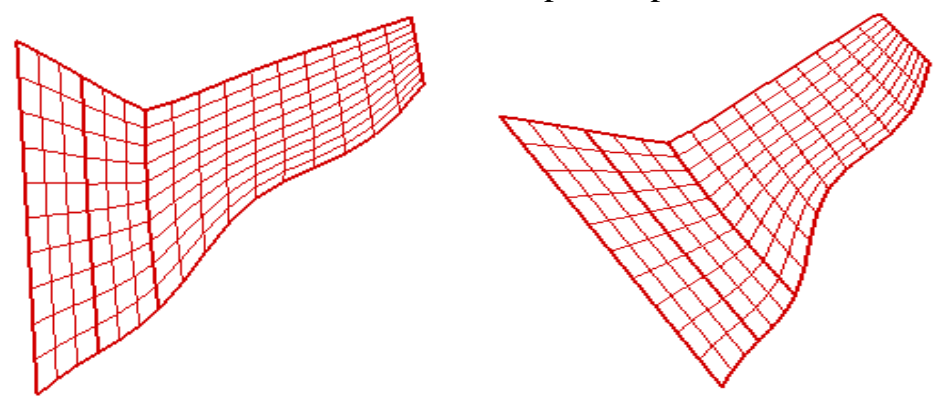

(f) The sixth mode shape comparison

Fig. 2 Mode shapes comparison (Left: present; Right: MSC.NASTRAN)

Afterwards, the mode shapes are calculated. The corresponding mode shapes are showed in Fig.2. The first mode is dominated by the first bending mode of component $\mathrm{C}$. The second mode is dominated by the second bending mode of component $\mathrm{C}$. The third mode is dominated by the first bending mode of component $\mathrm{B}$ and the first torsion of component $\mathrm{C}$. As for the last three modes, it is not easy to tell the dominated shape. For the fourth mode shape, the shape is apposite from the one via MSC.NASTRAN. To some extend, the mode shape comparison is good.

\section{Conclusion}

In this paper, a modified interface component mode synthesis of a folding wing is proposed based on fixed interface and loaded interface method. The efficacy is verified. It has been clearly demonstrated that the present method has a good accuracy. The inherent advantage of this method is reduction in size of problem. This method is a good choice when the interfaces have too many degrees of freedom. Finally, it can be employed to perform the transient dynamic analysis during the morphing process with less elastic coordinates. 


\section{Reference}

[1] Wilson J R. Morphing UAVs Change the Shape of Warfare.Aerospace America ,42(2004):28 29.

[2] Sofla A Y N , Meguid S A, Tan K T, Yeo W K.Shape morphing of aircraft wing:Status and challenges.Materials and Design, (2010)1284 1292.

[3] Gregory W. Reich, Jason C.Bowman, Brian Sanders. Development of an Integrated Aeroelastic Multibody Morphing Simulation Tool. 47th AIAA/ASME/ASCE/AHS/ASC Structures, Structural Dynamics, and Materials Conference. Rhode Island, (2006)2006 1892.

[4] John N.Scarlett, Robert A. Canfield, Brian Sanders. Multibody Dynamic Aeroelastic Simulation of a Folding Wing Aircraft. 47th AIAA/ASME/ASCE/AHS/ASC Structures, Structural Dynamics, and Materials Conference. Rhode Island, (2006)2006 213.

[5] Liu D.D, Chen P.C, Zhang. Z, Wang Z, Yang S. Lee. D.H. et al. Continuous Dynamic Simulation for Morphing Wing Aeroelasticity. 50th AIAA/ASME/ASCE/AHS/ASC Structures, Structural Dynamics, and Materials Conference. California,( 2009)2009 2572.

[6] Evgeny Selitrennik, Moti Karpel, Yuval Levy. Computational Aeroelastic Simulation of Rapidly Morphing Air Vehiclels.Journal of Aircraft. 49 (2012):1675 1686.

[7] Youghui Zhao, Haiyan Hu. Parameterized aeroelastic modeling and flutter analysis for a folding wing. Journal of Sound and Vibration.331(2012)308 324.

[8] Zhao Y.H. Hu H.Y. Prediction of transient responses of a folding wing during the morphing process. Aerospace Science and Technology. 24(2013):89 94.

[9] Roy R. Craig. Coupling of substructures for dynamic analysis: an overview. 47th AIAA/ASME/ASCE/AHS/ASC Structures, Structural Dynamics, and Materials Conference. Atlanta. (2000)2000 1573.

[10] R. R. Craig Jr., M.C.C. Bampton. Coupling of substructures for dynamic analyses.AIAA Journal.(1968)1313 1319.

[11] A. Shanmugam, Chandramouli Padmanabhan. A fixed-free interface compoent mode synthesis method for rotordynamic analysis.Journal of sound and vibration.(2006)664 679.

[12] Walter C. Hurty. Dynamic Analysis of Strctural Systems Using Component Modes.AIAA Journal (1965) 678 685.

[13] L.E. Suarez, M.P. Singh. Impoved Fixed Interface Method for Modal Synthesis. AIAA Journal. (1992)2952 2958.

[14] Daniel J. Rixen. A dual Craig-Bampton method for dynamic substructuring[J].Journal of computational and applied mathematics.(2004)168:383 391.

[15] W.A. Benfield, R. F. Hruda. Vibration Analysis of Strucutres by Component Mode Substitution. AIAA Journal. (1971)1255 1261.

[16] S.P. Lim, A. Q. Liu, K. M. Liew. Dynamics of flexible multibody systems using loaded-interface substructure synthesis approach.Computional Mechanics.(1994)15:270 283. 